\title{
Calculation of the metal reflectivity with taking polarization into consideration
}

\author{
O.V. Vakulenko ${ }^{1}$, V.S. Severin ${ }^{2}$ \\ ${ }^{1}$ Taras Shevchenko Kyiv National University, Physics Department, Chair of optics \\ 6, Academician Glushkov prospect, 03127 Kyiv, Ukraine \\ ${ }^{2}$ National Aviation University, Chair of theoretical physics \\ 1, Cosmonaut Komarov prospect, 03058 Kyiv, Ukraine
}

\begin{abstract}
Optical properties of free electrons in the conduction band of metal are considered. It is shown that the conventional Drude theory does not take shielding of the external electrical field by mobile electrons into account. This shielding is conditioned by the polarization of these electrons. Offered is the way of taking this polarization into consideration when calculating the reflectivity of metal. It is shown that the account of polarization results in agreement between the theoretical results and experimental data.
\end{abstract}

Keywords: polarization, electrical conductivity, optical constants, reflection spectra.

Manuscript received 03.11.06; accepted for publication 26.03.07; published online 01.06.07.

\section{Introduction}

The skin effect influences on reflection and absorption of light by metals [1]. It influences upon the optical behavior of noble and other metals with the high electrical conductivity (such as $\mathrm{Cu}, \mathrm{Al}$ ). In this case, the depth of the skin layer $\delta$ and the length of free path of an electron $l$ meet the condition $\delta<l$. If the electrical conductivity is enough small and the condition $\delta>l$ takes place, the optical behavior of metals is studied similarly to that of dielectrics and semiconductors. And in the spectral region that corresponds to light absorption by mobile electrons, the Drude theory is traditionally used [2-5].

The latter case $(\delta>l)$ is studied in this work. In the second section, it is shown that the conventional Drude theory neglects the shielding of the external electrical field (that is the polarization) by mobile electrons. The way to take this shielding into consideration is given in the third section. Light reflection is reviewed with taking the polarization into account in the fourth section. The comparison of the theoretical and experimental data is performed in the fifth and the sixth sections.

\section{The permittivity in the Drude model}

The optical properties of metals are dictated by its permittivity $\varepsilon$. The Drude model is traditionally used to determine $\varepsilon$ in the considered range of light frequencies $\omega$ [2-5]. In this model, free electrons (limited by the conduction band) are supposed to be neutralized by a positive background of a crystal lattice that has the permittivity $\varepsilon_{0}$ independent of frequency $[1,4,5]$. Thus, under action of the external electrical field $\mathbf{D}$ applied to metal there is an electric current with the density $\mathbf{j}=e n \mathbf{v}$, where $e$ is the charge of an electron, $\boldsymbol{v}$ is its speed, $n$ is the concentration of mobile electrons. The external field $\mathbf{D}(\omega) \exp (i \omega t)$ (where $\mathbf{D}(\omega)$ is the amplitude of the field) creates the current of these electrons with the amplitude of the electric current density $\mathbf{j}(\omega)$. The linear response of this current to the external field $\mathbf{D}$ is described by the expression

$\mathbf{j}(\omega)=s(\omega) \mathbf{D}(\omega) \frac{1}{\varepsilon_{0}}$,

where

$s(\omega)=\frac{n e^{2} \tau}{m(1+i \omega \tau)}$

is the electrical conductivity of the Drude model, $\tau=1 / \gamma$ is the relaxation time of the electron momentum, $m$ is its effective mass.

The permittivity of the kind of

$\varepsilon_{\mathrm{S}}(\omega)=\varepsilon_{0}+\frac{4 \pi}{i \omega} s(\omega)$

is traditionally used in the consideration of optical behavior of metals [2-5]. Eqs (2), (3) give the permittivity $\varepsilon_{s}$, its real part $\varepsilon_{s 1}=\operatorname{Re} \varepsilon_{s}$ and its imaginary one $\varepsilon_{\mathrm{s} 2}=\operatorname{Im} \varepsilon_{\mathrm{s}}$ as follows 
$\varepsilon_{S}(\omega)=\varepsilon_{0}\left(1-\frac{\omega_{p}^{2}}{\omega(\omega-i \gamma)}\right)$

$\varepsilon_{s 1}(\omega)=\varepsilon_{0}\left(1-\frac{\omega_{p}^{2}}{\omega^{2}+\gamma^{2}}\right)$,

$\varepsilon_{s_{2}}(\omega)=\varepsilon_{0} \frac{\omega_{p}^{2}}{\omega} \frac{\gamma}{\omega^{2}+\gamma^{2}}$,

where $\omega_{p}^{2}=\frac{4 \pi n e^{2}}{\varepsilon_{0} m}$ is the plasma frequency squared.

The Drude model can be applied only in the frequency region that is essentially larger than $\omega_{p}$ and cannot be used under decreased frequencies. It takes place because the shielding of the external field by mobile electrons in the Drude model is absent. This shielding starts to act when $t_{p}=\omega_{p}^{-1}$ [6]. Therefore, at times $t \approx t_{p}$ (that is at light frequencies $\omega \approx \omega_{p}$ ) the conduction electrons are exposed to the internal field $\mathbf{E}$. This field is $\mathbf{E} \neq \mathbf{D} / \varepsilon_{0}$. That is, the internal field $\mathbf{E}$ is not equal to the field $\mathbf{D} / \varepsilon_{0}$ that is the external field in relation to the conduction electrons. The polarization vector

$\mathbf{P}=\left(\mathbf{D}-\varepsilon_{0} \mathbf{E}\right) /(4 \pi)$

gives the difference $\mathbf{E}$ from $\mathbf{D} / \varepsilon_{0}[4,6-11]$. This difference is not taken into account in the obtained expressions (4)-(6) for the permittivity $\varepsilon_{s}$.

\section{Inclusion of polarization into the permittivity}

The current density $\mathbf{j}$ can be expressed both via the internal electrical field $\mathbf{E}$ and the field $\mathbf{D} / \varepsilon_{0}[6,7,9-11]$

$\mathbf{j}(\omega)=\sigma(\omega) \mathbf{E}(\omega)=s(\omega) \mathbf{D} / \varepsilon_{0}$.

The electrical conductivity $s$ determines the response of current to the external (in relation to the subsystem of conduction electrons of the metal) field $\mathbf{D} / \varepsilon_{0}$. And the electrical conductivity $\sigma$ gives the response of current to the internal field $\mathbf{E}$. Therefore, $s$ is named the external electrical conductivity, while $\sigma$ is named as the internal one [9-11]. The difference between $\sigma$ and $s$ in Exp. (7) appears when the polarization $\mathbf{P}$ differs from zero. The charges that do not participate in optical transitions in the studied range of frequencies create the background contribution $\varepsilon_{0}$ independent of frequency to the permittivity $[1,4,5,8]$. So, the expression for permittivity of metal $\varepsilon(\omega)$ can be written as follows

$\varepsilon(\omega)=\varepsilon_{0}+\frac{4 \pi}{i \omega} \sigma(\omega)$.
Eq. (8) stems from $\mathbf{D}(\omega)=\varepsilon(\omega) \mathbf{E}(\omega)$ that is the definition of permittivity of matter $\varepsilon(\omega)$, and $\mathbf{P}=(\mathbf{D}-$ $\left.\varepsilon_{0} \mathbf{E}\right) /(4 \pi)$ for the polarization vector $\mathbf{P}[4,6-11]$. But this definition requires $\sigma$ being in Eq. (8) instead of $s$ [6-11]. From this definition of $\varepsilon(\omega)$ and Eqs (7), (8) we deduce

$\sigma(\omega)=s(\omega) \varepsilon(\omega) \frac{1}{\varepsilon_{0}}$

And the formulas (8), (9) give

$\sigma(\omega)=\frac{1}{1-\frac{4 \pi}{i \omega \varepsilon_{0}} s(\omega)} s(\omega)$

Substituting Eq. (10) into (8), we obtain

$$
\varepsilon(\omega)=\varepsilon_{0} \frac{1}{1-\frac{4 \pi}{i \omega \varepsilon_{0}} s(\omega)}
$$

The precise expression for permittivity $\varepsilon(\omega)(11)$ (in which the polarization is taken into account) differs essentially from the approximated expression for the permittivity $\varepsilon_{s}(3)$ (in which the polarization is not taken into account and which is used by the conventional theory of optical behavior of metals). Developing the right side in the expression (11) as series in $s(\omega)$, we see that $\varepsilon(\omega)$ coincides with $\varepsilon_{s}(3)$ only when $s(\omega)$ is sufficiently small. Expression (10) allows us, under consideration of the optical behavior of metals (which are determined by the electrical conductivity $\sigma$ ), to use the electrical conductivity $s$ (obtained without regard for the polarization).

\section{Inclusion of polarization into the optical behavior of metals}

Substituting the expression (2) in the formula (11), we have

$$
\varepsilon(\omega)=\varepsilon_{0} \frac{1}{1+\frac{\omega_{p}^{2}}{\omega(\omega-i \gamma)}} .
$$

Exp. (12) gives the real part $\varepsilon_{1}=\operatorname{Im} \varepsilon$ and the imaginary part $\varepsilon_{2}=-\operatorname{Im} \varepsilon$ as

$$
\varepsilon_{1}(\omega)=\varepsilon_{0} \frac{\gamma^{2}+\omega^{2}\left(1+\frac{\omega_{p}^{2}}{\omega^{2}}\right)}{\gamma^{2}+\omega^{2}\left(1+\frac{\omega_{p}^{2}}{\omega^{2}}\right)^{2}} \equiv \varepsilon_{0}\left[1-\frac{\omega_{p}^{2}\left(1+\frac{\omega_{p}^{2}}{\omega^{2}}\right)}{\gamma^{2}+\omega^{2}\left(1+\frac{\omega_{p}^{2}}{\omega^{2}}\right)^{2}}\right]
$$




$$
\varepsilon_{2}(\omega)=\varepsilon_{0} \frac{\omega_{p}^{2}}{\omega} \frac{\gamma}{\gamma^{2}+\omega^{2}\left(1+\frac{\omega_{p}^{2}}{\omega^{2}}\right)^{2}}
$$

Making expansion of the right side of expressions (12)-(14) in series by degrees of $\left(\omega_{p} / \omega\right)^{2}$, we will be convinced in the validity of the ratio

$\varepsilon(\omega)=\varepsilon_{\mathrm{s}}(\omega)+O\left(\left(\frac{\omega_{p}}{\omega}\right)^{4}\right)$.

Hence, the permittivity $\varepsilon_{S}(\omega)$ (in which the polarization is not taken into account) can be applicable only in the approximation of large frequencies $\omega^{2}>>\omega_{p}^{2}$.

Let's consider optical constants of metal as a function of the light wavelength $\lambda=2 \pi c / \omega$, where $c$ is the speed of light. Let's designate the plasma wavelength as $\lambda_{p}=2 \pi c / \omega_{p}, \lambda_{1}=2 \pi c \tau, z=\lambda_{1} / \lambda_{p}$. In new notations, we have from formulas (5), (6)

$$
\begin{gathered}
\varepsilon_{s 1}=\varepsilon_{0}\left[1-\frac{z^{2}}{1+\left(z \frac{\lambda p}{\lambda}\right)^{2}}\right], \\
\varepsilon_{S 2}=\varepsilon_{0} z \frac{\lambda / \lambda_{p}}{1+\left(z \frac{\lambda_{p}}{\lambda}\right)^{2}} .
\end{gathered}
$$

And from the expressions (13), (14), we have

$$
\begin{gathered}
\varepsilon_{1}=\varepsilon_{0}\left\{1-\frac{z^{2}\left[1+\left(\frac{\lambda}{\lambda_{p}}\right)^{2}\right]}{1+\left(z \frac{\lambda_{p}}{\lambda}\right)^{2}\left[1+\left(\frac{\lambda}{\lambda_{p}}\right)^{2}\right]^{2}}\right\}, \\
\varepsilon_{2}=\varepsilon_{0} z \frac{\lambda / \lambda_{p}}{1+\left(z \frac{\lambda_{p}}{\lambda}\right)^{2}\left[1+\left(\frac{\lambda}{\lambda_{p}}\right)^{2}\right]^{2}} .
\end{gathered}
$$
is [3]

The reflectivity of metal for normal light incidence

$$
R(\lambda)=\frac{(N(\lambda)-1)^{2}+K(\lambda)^{2}}{(N(\lambda)+1)^{2}+K(\lambda)^{2}} .
$$

Here,

$$
K(\lambda)=\sqrt{\frac{1}{2}\left(-\varepsilon_{1}+\sqrt{\varepsilon_{1}^{2}+\varepsilon_{2}^{2}}\right)}
$$

is the index of absorption,

$$
N(\lambda)=\sqrt{\frac{1}{2}\left(\varepsilon_{1}+\sqrt{\varepsilon_{1}^{2}+\varepsilon_{2}^{2}}\right)}
$$

is the refraction index, and $\varepsilon_{1}, \varepsilon_{2}$ are given by the formulas (17), (18). Exp. (19) considers the polarization. Without regard for polarization, the light reflectivity of metal is

$$
R_{s}(\lambda)=\frac{\left(N_{s}(\lambda)-1\right)^{2}+K_{s}(\lambda)^{2}}{\left(N_{s}(\lambda)+1\right)^{2}+K_{s}(\lambda)^{2}},
$$

where

$$
\begin{aligned}
& K_{S}(\lambda)=\sqrt{\frac{1}{2}\left(-\varepsilon_{s 1}+\sqrt{\varepsilon_{s 1}^{2}+\varepsilon_{s 2}^{2}}\right)}, \\
& N_{s}(\lambda)=\sqrt{\frac{1}{2}\left(\varepsilon_{s 1}+\sqrt{\varepsilon_{s 1}^{2}+\varepsilon_{s 2}^{2}}\right)},
\end{aligned}
$$

and $\varepsilon_{s 1}, \varepsilon_{s 2}$ are given by the formulas (15), (16).

\section{Comparison of the theory with experiment}

Let's try to match the obtained theoretical results with experimental data for the normal light reflectivity of metal.

In Figs 1-3, the experimental values of normal light incidence reflectivity for polycrystalline metals $\mathrm{Ni}$ [12], $\mathrm{Fe}$ [13], $\mathrm{Nb}$ [14] are given by circles. In these figures, the dotted line represents results of the calculation of the reflectivity by using the formula (22), which corresponds to the conventional Drude theory and not takes the polarization into account. In the same figures, the continuous line gives results of the calculation for normal light incidence reflectivity in accord with the formula (19), which accounts for polarization.

The values of the physical quantities $n, \varepsilon_{0}$, $\xi=m / m_{0}$ (where $m_{0}$ is the electron mass), used in the calculation, are given in the table. The value $n$ was determined as the product of the metal valence (equal to 2 ) by the concentration of atoms of metal (taken from tab. 1.5 from [4]).

Table.

\begin{tabular}{|cccl|}
\hline Metal & $n \cdot 10^{-22}, \mathrm{~cm}^{-3}$ & $\varepsilon_{0}$ & $\xi$ \\
\hline $\mathrm{Ni}$ & 18.28 & 0.85 & 1.4 \\
\hline $\mathrm{Fe}$ & 17.00 & 0.95 & 2.5 \\
\hline $\mathrm{Nb}$ & 11.12 & 0.45 & 1.8 \\
\hline
\end{tabular}



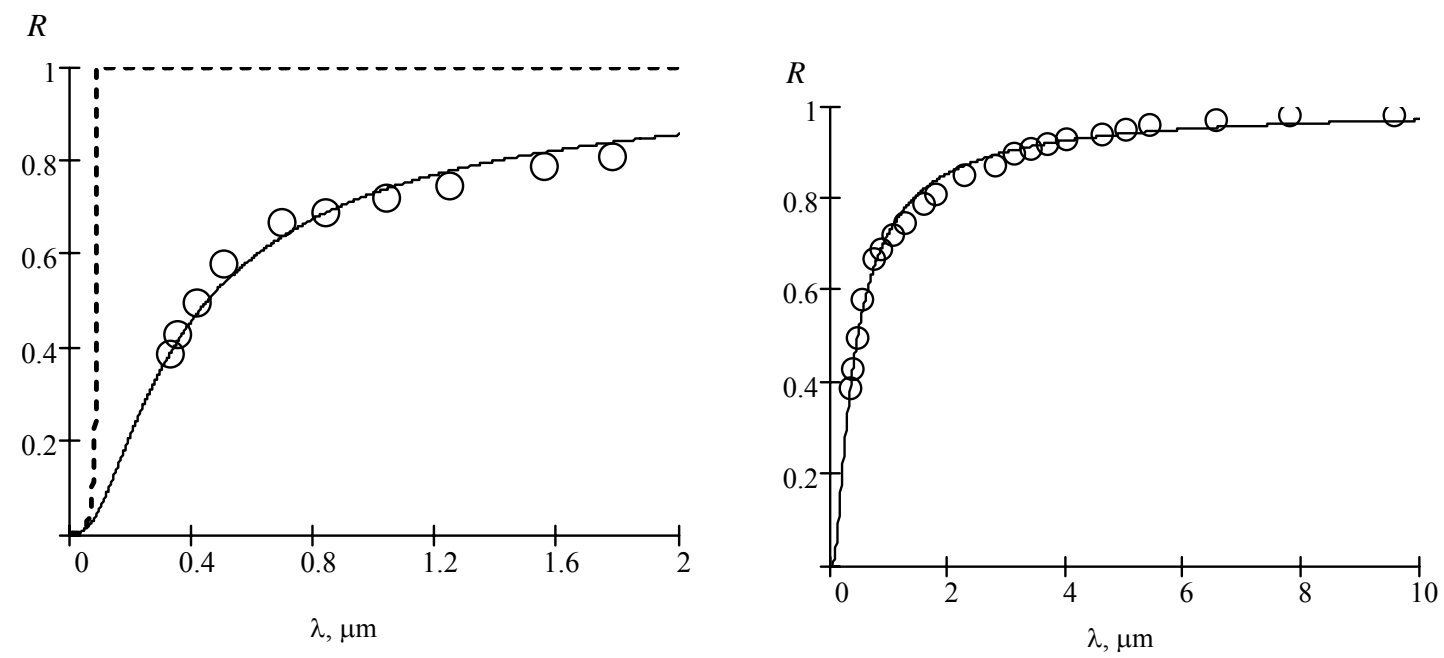

Fig. 1. The dependence of the normal light incidence reflectivity of metal $R$ at the wavelength $\lambda$ for Ni. The circles are experimental values, the dotted line is the calculation by the formula (22) and the continuous line is the calculation by the formula (19).
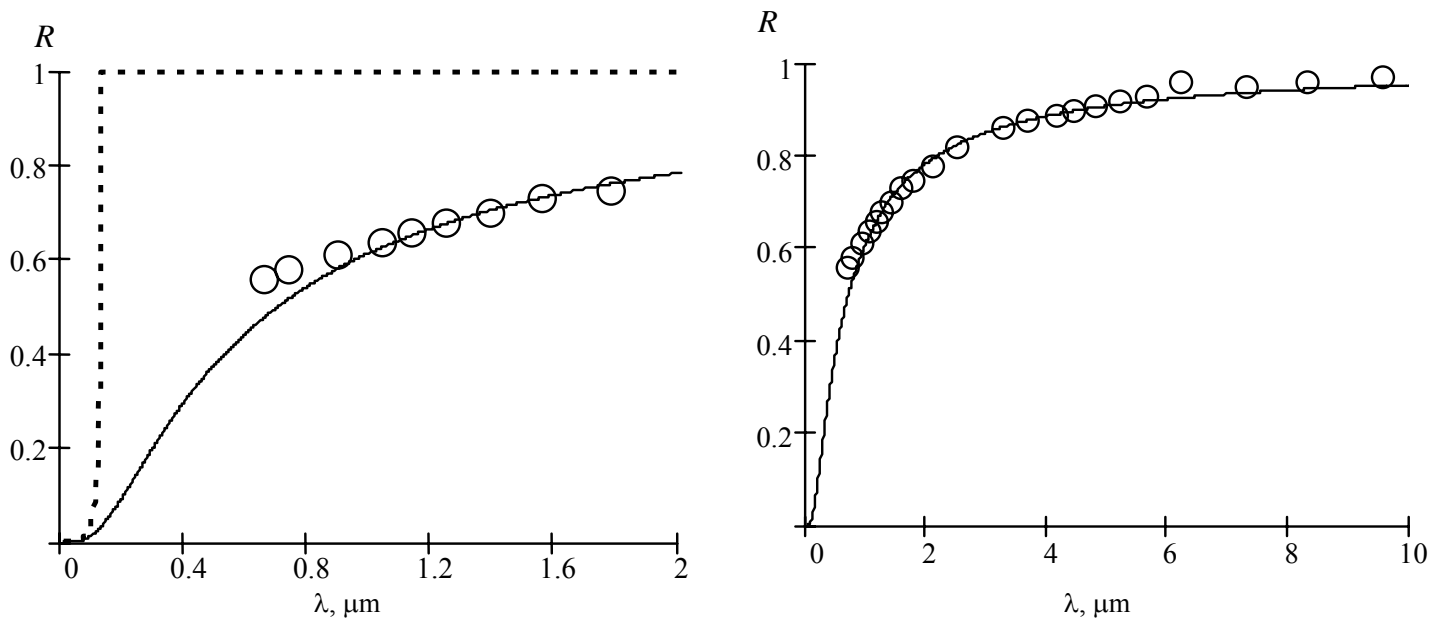

Fig. 2. The dependence of normal light incidence reflectivity of metal $R$ at the wavelength $\lambda$ for Fe. The designations are the same as in Fig. 1.
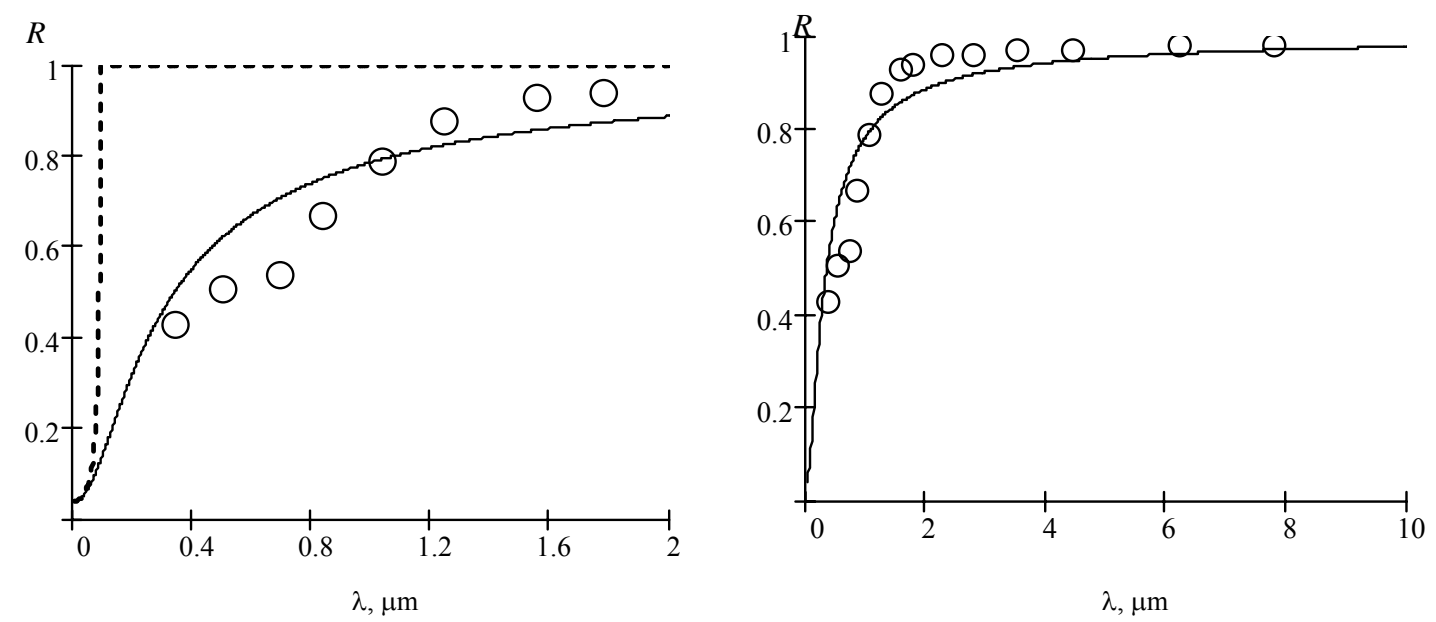

Fig. 3. The dependence of normal light incidence reflectivity of metal $R$ at the wavelength $\lambda$ for $\mathrm{Nb}$. The designations are the same as in Fig. 1. 
The values of $\varepsilon_{0}$ and $\xi$ were fitting parameters. The calculation was performed at $\tau=10^{-12} \mathrm{~s}$. Within the range $\lambda>\lambda_{p}$, the variation $\tau$ was within $10^{-12}-10^{-14} \mathrm{~s}$ and did not influence the results of calculating the reflectivity by the formula (19).

\section{Discussion}

It can be seen from figures that the reflectivity for normal light incidence computed with and without the allowance for polarization coincides only at small $\lambda$ (that is, in the approximation of large frequencies $\omega>>\omega_{p}$ ) as it should be. As $\lambda$ increases (it can be seen from figures) the result of computation of the normal light incidence reflectivity by the formula (22) (which uses the conventional Drude theory and does not consider polarization) does not correspond to experimental data at all and differs from them dramatically. However, the result of computation for the normal light incidence reflectivity by the formula (19) (which allows for polarization) gives the coincidence with experimental data at this $\lambda$.

Thus, the abovementioned way of taking polarization into account gives a good agreement between the theoretical results and experimental data.

\section{References}

1. 1. M.N. Libenson, Optics of metals. Fizicheskaya entsiklopediya. Sov. entsiklopediya Publ., Moscow, 1992. V. 3, p. 110-112 (in Russian).
2. P. Grosse, Free electrons in solids. Mir Publ., Moscow, 1982 (in Russian).

3. Yu.I. Ukhanov, Optical properties of semiconductors. Nauka, Moscow, 1977 (in Russian).

4. Ch. Kittel, Introduction to solid-state physics. Nauka, Moscow, 1978 (in Russian).

5. Yu. Madelung, Theory of solids. Nauka, Moscow, 1980 (in Russian).

6. F. Platzman, P. Volf, Waves and interactions in plasma of solids. Mir, Moscow, 1975 (in Russian).

7. Yu.A. Il'inskiy, L.V. Keldysh, Interaction of electromagnetic radiation with matter. Tutorial. Moscow State University Publ., Moscow, 1989 (in Russian).

8. S.I. Pekar, Optics of crystals and additional light waves. Naukova dumka, Kiev, 1982 (in Russian).

9. R. Kubo, The fluctuation-dissipation theorem // Repts Progr. Phys. 29, Pt. 1, p. 255-284 (1966).

10. T. Izuyma, An expansion theorem for the electric conductivity of metals. I. Electric conductivity for longitudinal electric field // Progr. Theor. Phys. 25 (6), p. 964-980 (1961).

11. R. Eykholt, Extension of the Kubo formula for the electric-conductivity tensor to arbitrary polarization of electric field // Phys. Rev. B 34 (10), p. 66696674 (1986).

12. D.W. Lynch, R. Rosei, J.H. Weaver // Solid State Communs 9, p. 2195 (1971).

13. J.H. Weaver, E. Colavita, D.W. Lynch, R. Rosei // Phys. Rev. B 19, p. 3850 (1979).

14. J.H. Weaver, D.W. Lynch, C.G. Olson // Phys. Rev. B 7, p. 4311 (1973). 